\title{
(72) SEISMIC BEHAVIOR OF A GROUPED-PILES SUPPORTED BRIDGE PIER
}

\author{
Hirokazu Takemiya \\ Department of Civil Engineering, Okayama University \\ Takashi Tazoh and Katsumi Shimizu \\ Institute of Technology, SHIMIZU Construction Co., Ltd.
}

\section{INTRODUCTION}

The authors have been engaged in the investigation of seismic response characteristics of pile-supported bridge structures. In the theoretical approach, they have developed different methods of analyses; A beam analysis in a visco-elastic medium $[1,2]$, a finite element procedure [3,4] and a Green function method [5]. All of the methods aim at evaluating the so-called pile head impedance functions and the associated seismic driving forces at the footing elevation. With use of these quantities, the component modes technique can be applied for the effective and efficient seismic analysis of the whole soil-structure interaction system.

The grouped pile foundations are characterized by the so-called the grouping effect due to the pile-soil-pile interaction. As stated in Refs.[1-5], in the case of a small number of piles, the rigorous analysis may be taken, while in the case of a large number of piles the approximate but effective procedure such as a ring-pile concept [3] is more desirable from the computational point of view. Some simplification may further be introduced to advantage for this aim, as proposed in Ref.[2].

In this paper, the authors extended the preceding formulation for vertical piles $[1,2]$ to the battered piles analysis. Since the foundation for analysis has a large number of piles, the ring-pile modeling is adopted with an equivalent independent soil stiffness at ring-pile nodes. For the purpose of verifying these assumptions, thus obtained results are compared with the earthquake observation data. The particular points of investigation are placed on the structural responses when the battered piles are used together with the vertical ones, and when the footing embedment is considered.

\section{THEORETICAL FORMULATION}

For the seismic analysis of a pile supported structure, the modeling is carried out as outlined below: (1) Soils are horizontal layers of visco-elastic materials with hysteretic damping whose properties remain constant within individual layer. (2) The pile foundation is comprised of a group of vertical and battered straight piles of circular cross sections, and a rigid body pile cap (or footing) into which pile heads are jointed. (3) For evaluating the lateral soil reaction around piles and footing, the infinetesimal thin layer or equivalently the plane strain assumption is introduced. The soil reaction at the footing bottom is neglected since its contribution is so small. The interaction force between soil and piles is accounted for by evaluating the soil sitffness as the inverse of the soil flexibility. A simple diagonalization as proposed in Ref.[2] is used for an efficient solution procedure. (4) Piles are modeled by the mass distributed beam elements with hysteretic damping. (5) The superstructure is discretized and formulated by the standard matrix structural analysis procedure. The normal modes decomposition is presumed for this part for the fixed base condition. (6) As for the seismic motion, the shear wave field is considered; therefore, the base input situation is dealt with. (7) For the seismic analysis of the concerned soil-structure, the substructure technique is applied to advantage, splitting it into soils, piles, footing and superstructure. The global interaction behavior of piles and soil in their vicinity is approximated by the so-called ringpile formulation $[2,3,4]$ which presumes limited Fourier harmonics expansion to express the response variation of pile axes on concentric rings. (9) As an engineering understanding, the pile head impedance functions and the associated driving forces, which are frequency dependent, are evaluated, Because of this nature, the inertial interaction analysis between the sub- and superstructures is carried out in the frequency domain. 


\section{Numerical Computation and Earthquake Observation}

Concerning the earthquake response of grouped piles supporting bridge structure, Ohira, Tazoh and Shimizu carried out earthquake observations during the period of 1981-1985 at a road bridge in Fujisawa, Kanagava Prefecture, Japan. This bridge is characterized by a three span continuous girder on medium height piers, as illustrated in Fig.1. The connection of the pier No.6 is rigid with the girder and other pier connections are movable along the bridge axis. The site is an extremely soft alluvial deposits of humus and silt, the average shear wave velocity being $57 \mathrm{~m} / \mathrm{sec}$, which lies on a firm diluvium of the shear velocity more than $400 \mathrm{~m} / \mathrm{sec}$. The observation system and the findings from it have been reported in detail in Ref.[6].

The structure for investigation herein is chosen as the above bridge pierfoundation system. Since the above earthquake observation is mainly addressed to the pier No.6, the present analysis is also focused on its response. This foundation is characterized by the battered outer piles together with the vertical centeral piles along the bridge axis. Note that a half of the total 64 piles are battered by $10^{\circ}$ from the vertical.

Pier-Foundation Modeling: The structural model for theoretical analysis is depicted in Fig. 2 and the soil condition used are indicated in Fig. 3. The soil profile is idealized as a layered system which extends infinitely in the horizontal direction, even if we consider the longitudinal behavior of the concerned bridge pier. Although the original grouped-pile foundation has equally spaced piles of 8 rows by 8 columns in the plan view at the pile head elevation, the piles are rearranged as symmetric piles with respect to $\mathrm{x}$ - and $\mathrm{y}$-axes on 9 rings. The pier and footing part is represented by a descrete 5-mass system. The top mass takes account of the 3-span continuous mass together with the contribution from the top segment of the pier.

In order to execute the parametric studies, the authors adopted the following four models, by changing the conditions of the footing and the piles: (1) All vertical piles (referred to simply as vertical piles in the computation results) and footing without embedment, (2) A mixed type of battered and vertical piles (simply referred to as the battered piles in the computation results) and footing without embedment, (3) A11 vertical piles and footing with embedment, and (4) A mixed type of battered and vertical piles and footing with embedment.

Pile Head Impedance Functions: Fig. 4 shows the gross pile heads impedance functions evaluated at the gravity center of the footing. The differences between the all vertical piles foundation and the battered and vertical piles foundation are recognized appreciably at the impedance functions for the rocking and strongly for its cross degree-of-freedom(DOF) with the sway. The real part of the impedance function of the battered and vertical piles results in a smaller value than that of the all vertical piles foundation for the rocking motion in the whole frequency range. Regarding the cross stiffness, we note that the battered and vertical piles foundation yields a positive value in contrast to the negative value of the all vertical piles foundation. This opposite sign in magnitude in battered piles implies the retrograde motion in which the horizontal translation due to the rocking moment makes a reduction of the horizontal movement due to the sway motion, diminishing the response at the superstructure. The embedment effect of the footing only results in a small modification of the impedance function for sway motion in the high frequency range.

Total Interaction for Seismic Motion: The response characteristics are interested with the emphasis on the soil-strurcture interaction. Fig.5(a) depicts the frequency responses for the case of the battered and vertical piles for a unit harmonic base motion at the pile tips elevation. The first peak, being almost identical for both footing and pier top, represntes the fundamental soil vibration mode (for vertical shear waves) which yields the sway mode to the structure. The second peak reflects the rocking mode of foundation due to the interaction with soils since we note that a small response at footing and appreciable amplification at the pier top. The second soil vibration mode, located at around this frequency works to increase the retorograde rocking motion for the battered piles foundation and gives a significant response reduction in horizontal motion at the footing elevation. In the figure, the pier top response is also depicted for the almost fixed condition at its footing. 
Actually this condition is produced by multiplying the pile head impedance functions by one thousand in magnitude. We note that the predominant peak around $2.6 \mathrm{~Hz}$ coincides the superstructural fundamental mode in which the girder part sways with all piers in a phase motion. This vibration mode disappears in the soil-structure interaction system. Rather, the trough at the footing elevation and the value nearby the second peak at the pier top at the $2 \mathrm{~Hz}$ mean a great pier motion in the form of rocking.

In case of all vertical piles, shown in Fig.5(b), the difference from the battered piles is noted such as the bigger first peak and the more reduction of the second peak. The former explains the greater input motion for the all vertical piles foundation and the latter is due to the no presence of the retrograde motion as seen in the battered piles foundation. In the higher frequency range, the repsonse of the battered piles are greater than that of the all vertical piles foundation.

Since the earthquake observation data are available for the structure of the present analysis, the comparison is made with the theoretical ones as the former being the bench mark for the parametric discussion. Fig. 6. shows the reponse amplification against the base input which is obtained from the records of the Kanagawa-YamanashiKenzakai Earthquake on August 8, 1983. This is the biggest earhtquake among those observed at the bridge and has the following properties: Magnitude $=6.0$, Epicentral distance $=42 \mathrm{~km}$, Epicentral depth $=20 \mathrm{~km}$, Maximum Acceleration at soil surface $=113.6$ gal. The observed amplification is simply calcurated by dividing the Fourier transform of observed accelerograms at fooing top and pier top by the value at $-30 \mathrm{~m}$ in soils. Concerning the piles, the battered analysis leads a good matching with the observation. The all vertical piles analysis fails to give the response matching at the second peak and the response features in the high freuqncy range as stated in the above paragraph.

The embedment effect is investigated by comparing the responses for foundations with it and without it. Their comparison with the observation results indicates that the consideration of the embedment, even under the present approxmate manner, explains well the response characteristics in the freuqncy range of interest. The embedment of the footing works for the increase of response at the second peak while for the reduction in the high freuqncy range beyond this freuqncy. The former is understood as the increase of the input motion due to the second soil vibration mode in favor of the retrograde motion of the battered piles foundation and the latter is as the stiffness effect for the foundation motion. The footing embedment changes the soil stiffness that results in the shift of the soil-structure interaction mode toward lower frequency range. The same trand holds also for the all vertical piles foundation.

\section{CONCLUSION}

The comparison of the analytical results with the earthquake observation data confirms that the present method is very effective. The investigation is focused on the effect of the battered piles and embedment of the footing on the superstructural response. The battered piles motion is noted to be retrograde in which the rotational motion of the foundation is to reduce the superstructural response due to the positive interaction of sway and rocking modes in contrast to the vertical piles foundation which is characterized by the prograde motion. Therefore, the modeling of the battered piles by the equivalent vertical piles will be limited in the response prediction. The embedment effect of the footing works first to amplify and then to reduce the response of the soil-structure interaction as the frequency grows beyond the fundamental soil vibration.

\section{REFERENCES}

1)Takemiya,H. and Yukawa,Y., "Dynamic Analysis of Grouped Pile Foundation in Layered Soils," Dtructural/Earthquake Eng., JSCE, Vol.3, No.1, April 1986(Proc.JSCE, No.368/I-5), pp.183s-193s.

2)Takemiya,H., Yukawa,Y. and Yabushita,J., "Response of Grouped Piles Foundation for Base input", Proc. of Symp. on Earthquake Engineering Research(Jishin Kogaku Kenkyu Hapyokai), JSCE, July, 1985(in Japanese), PP.193-196.

3)Takemiya,H., "Ring-Pile Analysis for a Grouped Pile Foundation Subjected to Base Motion," Structural/Earthquake Eng., JSCE, Vol.3, No.1, April 1986 (Proc.JSCE, No.368/I-5), pp.195s-202s. 4) Takemiya,H. and Hiramatsu,E., "3-D FEM Dynamic Analysis System for Embedded Structures," JSCE, (Proc. of Symp. on Earthquake Engineering Research(Jishin Kogaku Kenkyu Hapyokai).

5)Takemiya,H. and Tanaka,H., "Method of Dynamic Analysis for A Grouped Pile Foundation and Its Dynamic Characteristics," JSSMFE, Annual Conf. 1987.6.

6) Ohira, A., Tazoh,T., Dewa, K., Shimizu,K. and Shimada,M., "Observations of Earthquake Response Behaviors of Foundation Piles for Road Bridge," Procs. of the 8th WCEE, San Francisco, Vol.III, July 1984, pp.577-584. 

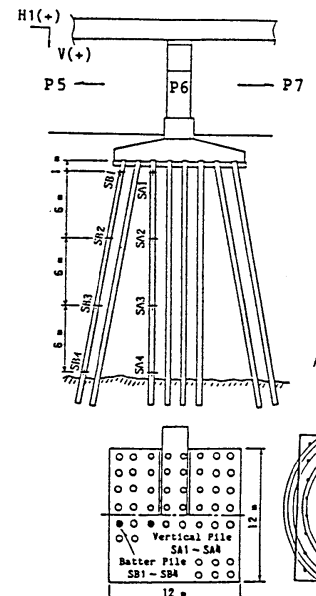

Fig.1 Bridge-Pier for Analysis
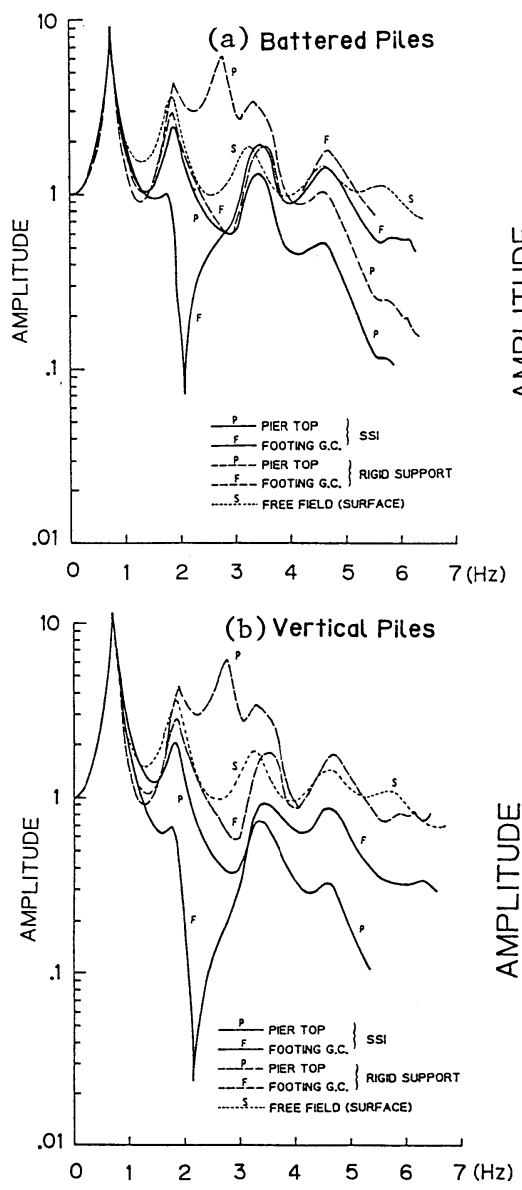

Fig. 5 Comparison of Response Amplifications
Fig. 2 Model for Computation
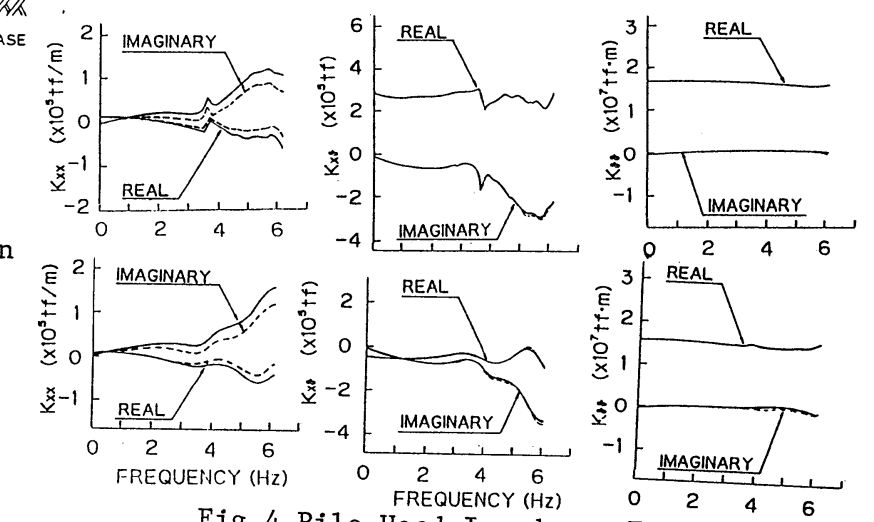

Fig. 4 Pile Head Impedance Functions $(\mathrm{Hz})$
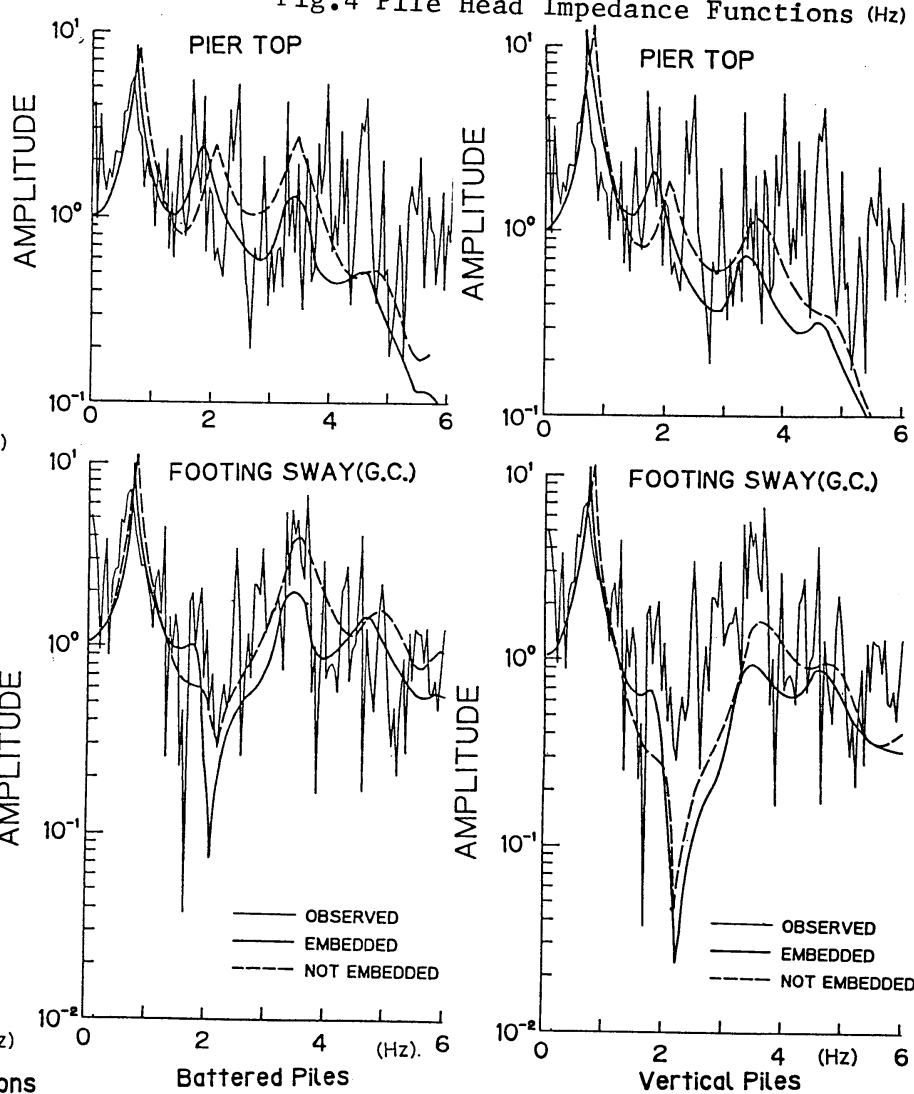

Fig.6. a Response Amplifications

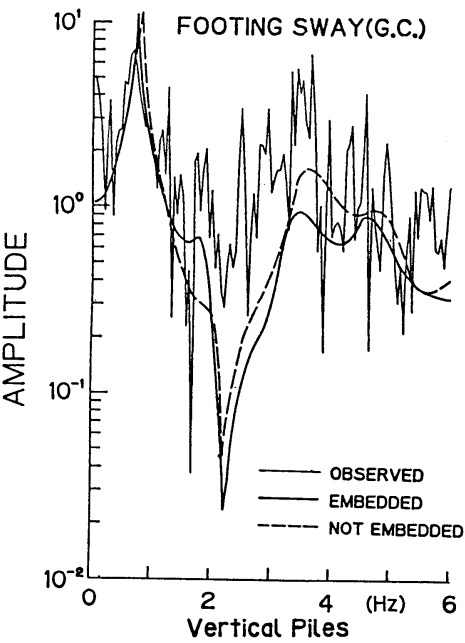

Fig.6.b Response Amplifications 